\section{BOOKBAG: A FORTRAN IV program for calculating various parameters of the "book bag and poker chips" Bayesian task}

\author{
AMNON TILL \\ Victoria University of Wellington \\ Private Bag, Wellington, New Zealand
}

In a typical experiment on Bayesian probability revision (e.g., Edwards, Lindman, \& Phillips, 1965), subjects are informed that there are two bags ( $\mathrm{H}$ and $\overline{\mathrm{H}})$ containing chips of two colors, say, red and blue. In $\mathrm{H}$, red chips predominate (e.g., 70 red and 30 blue), whereas in the other bag, blue chips predominate (e.g., 30 red and 70 blue). One of the bags is chosen randomly by the experimenter, with probability of choice for each bag equal to half. The task of the subject is to look at the sample chips (one or more chips, sampled with replacement) that the experimenter picked from one of the bags and to guess (by stating a probability estimate) from which of the bags $(\mathrm{H}$ or $\widetilde{\mathrm{H}})$ the sample was taken.

The normative model. The normative Bayesian model for this task is calculated in the following way:

$$
\begin{aligned}
& P(H \mid D)=\frac{P(D \mid H) P(H)}{P(D \mid H) P(H)+P(D \mid \bar{H}) P(\bar{H})} \\
& P(\bar{H} \mid D)=\frac{P(D \mid \bar{H}) P(\bar{H})}{P(D \mid H) P(H)+P(D \mid \bar{H}) P(\bar{H})} .
\end{aligned}
$$

We divide Equation 1 by Equation 2:

$$
\frac{\mathrm{P}(\mathrm{H} \mid \mathrm{D})}{\mathrm{P}(\overline{\mathrm{H}} \mid \mathrm{D})}=\frac{\mathrm{P}(\mathrm{D} \mid \mathrm{H}) \mathrm{P}(\mathrm{H})}{\mathrm{P}(\mathrm{D} \mid \overline{\mathrm{H}}) \mathrm{P}(\overline{\mathrm{H}})}
$$

We can term the left side of Equation 3 posterior odds or $\Omega$ i. Also, we can term $P(D \mid H) / P(D \mid \bar{H})$ the likelihood ratio, $L R$, and term $P(H) / P(\bar{H})$ the prior odds, $\Omega$ o.

So we can rewrite Equation 3 in the following way:

$$
\Omega \mathrm{i}=\Omega \text { o.LR. }
$$

In this task the prior probabilities of $P(H)$ and $P(\bar{H})$ are equal; therefore,

$$
\frac{\mathrm{P}(\mathrm{H})}{\mathrm{P}(\overline{\mathrm{H}})}=\frac{.5}{.5}=1
$$

Since $\Omega o=1$,

$$
\Omega \mathrm{i}=\mathrm{LR} .
$$

In most psychological studies of this kind, the bag composition is symmetrical (as in our example above). Then,

$$
\Omega i=L R=\left(\frac{p}{1-p}\right)^{r-b}
$$

(see Edwards et al., 1965) where $\mathrm{p}$ is the proportion (or probability) of red chips in bag $\mathrm{H}, \mathrm{r}$ is the number of red chips in the sample, and $b$ is the number of blue chips in the sample. Because of the symmetry in the compositions of the two bags, $1-p$ is equal to the probability of red chips in the $\overline{\mathbf{H}}$ bag.

The program. Bookbag is a program designed to facilitate the calculation of the various parameters in the "book bag and poker chips" task. It can be used in several ways; for example, for teaching students this type of task or as a subroutine in a bigger program that may provide the subjects with feedback on the various normative solutions to this problem.

Input. The program varies (1) the number of red chips in the two bags, $H$ and $\overline{\mathbf{H}}$ (or alternatively the values of $p$ and $1-p$ ) and (2) the exponent $r-b$, or the number of red and blue chips in the sample.

Output. The program calculates for each combination of $p$ and $1-p$ and for $r-b$ values (1) the posterior odds $\Omega \mathbf{i},(2)$ the log base 10 of the posterior odds, and (3) the posterior probability $\mathrm{P}(\mathrm{H} \mid \mathrm{D})$.

Restrictions. The program is written in FORTRAN IV so any computer system which supports FORTRAN IV will run it. It takes about $1.5 \mathrm{sec}$ to run this program on the CYBER 73 computer.

Availability. A copy of the listing, documentation, and sample of output can be obtained free of charge by writing to Amnon Till, Department of Psychology, Victoria University of Wellington, Private Bag, Wellington, New Zealand.

\section{REFERENCES}

Edwardos W., Lindman, H., \& Phillips, L. D. Emerging technologies for decision making. In New directions in psychology 2. New York: Holt, 1965. 